\title{
A SUBPOPULATION OF ASTROCYTE PROGENITORS DEFINED BY SONIC HEDGEHOG SIGNALING
}

AUTHORS: Ellen Gingrich ${ }^{1}$, Kendra Case ${ }^{2}$, A. Denise R. Garcia ${ }^{2,3^{*}}$

'Department of Biology, Stanford University, Stanford, CA 94305

${ }^{2}$ Drexel University, Philadelphia, PA 19104, USA

${ }^{3}$ Department of Neurobiology and Anatomy, Drexel University College of Medicine, Philadelphia, PA 19129, USA

${ }^{*}$ Corresponding author

\section{Author contributions}

Conceptualization, E.G. and A.D.R.G., Investigation, E.G., K.C., A.D.R.G., Writing, E.G., A.D.R.G., Funding Acquisition, A.D.R.G.

Correspondence:

A. Denise R. Garcia

Department of Biology

Department Neurobiology and Anatomy

Drexel University

3245 Chestnut St. PISB 422

Philadelphia, PA 19104

adg82@drexel.edu 


\section{ABSTRACT}

2 The molecular signaling pathway, Sonic hedgehog (Shh), is critical for the proper development

3 of the central nervous system. The requirement for Shh signaling in neuronal and

4 oligodendrocyte development in the developing embryo are well established. Here, we show

5 that Shh signaling also operates in a subpopulation of progenitor cells that generate cortical

6 astrocytes. In the neonatal brain, cells expressing the Shh target gene, Gli1, are found in the

7 subventricular zone (SVZ), a germinal zone harboring astrocyte progenitor cells. Using a

8 genetic inducible fate mapping strategy, we show that these cells give rise to half of the

9 cortical astrocyte population, suggesting that the cortex harbors astrocytes from different

10 lineages. Shh activity in SVZ progenitor cells is transient but recurs in a subpopulation of

11 mature astrocytes localized in layers IV and V in a manner independent of their lineage. These

12 data identify a novel role for Shh signaling in cortical astrocyte development and support a

13 growing body of evidence pointing to astrocyte heterogeneity. 


\section{INTRODUCTION}

Astrocytes encompass a diverse population of cells that possess a broad array of

functional properties that are essential for nervous system function. The developmental

17 processes that confer their functional characteristics are not well understood. While a large body of work has delineated molecular mechanisms underlying astrocyte specification during embryonic development, the processes surrounding postnatal astrocyte development, the time during which most astrocytes are generated, are considerably less well defined.

The Sonic hedgehog (Shh) signaling pathway is best characterized during embryonic neurodevelopment, where it exerts powerful influence over a broad array of neurodevelopmental processes, including patterning and morphogenesis, axon pathfinding,

24 and cell type specification of ventral motor neurons and oligodendrocytes ${ }^{1}$. However, in the adult brain canonical Shh activity is found predominantly in astrocytes ${ }^{2,3}$. Little is known about the role of Shh signaling in astrocyte development. In the developing optic nerve, SHH derived

27 from retinal ganglion cells promotes proliferation of astrocyte precursors ${ }^{4}$. In contrast, $\mathrm{SHH}$ in

28 the early embryonic spinal cord limits the specification of astrocyte progenitor cells ${ }^{5}$. Whether Shh signaling plays a role in cortical astrocyte development, is not known.

Astrocyte production occurs primarily during the first two weeks of postnatal development ${ }^{6}$. They are derived from radial glia and progenitor cells in the ventricular zone (VZ) and subventricular zone (SVZ) $)^{7-11}$, respectively, as well as from local proliferation of

33 differentiated astrocytes ${ }^{12}$. The dorsal region of the SVZ harbors progenitor cells that

34 generate both oligodendrocytes and astrocytes ${ }^{8,13}$. The postnatal ventricular and

35 subventricular zones (V-SVZ) harbors a population of progenitor cells that express Gli1, a

36 transcriptional target of Shh signaling ${ }^{14,15}$. Gli1-expressing progenitors generate a substantial

37 population of oligodendrocytes that populate the overlying white matter ${ }^{13}$. However, in the 
mature cortex, a discrete subpopulation of astrocytes express Gli1, reflecting high levels of

Shh activity ${ }^{3}$. Astrocyte transduction of SHH occurs predominantly in cells residing in layers IV and $\mathrm{V}^{16}$, consistent with the localization of $\mathrm{SHH}$ in layer $\mathrm{V}$ pyramidal neurons ${ }^{3,17}$. The precise relationship between Gli1 progenitors in the postnatal SVZ and transduction of SHH in mature astrocytes is not known. One possibility is that superficial and deep layer astrocytes are derived from distinct progenitor pools, with Gli1 precursors exclusively generating deep layer astrocytes. Indeed, large scale transcriptomic analysis of the postnatal cortex shows that astrocytes in deep cortical layers exhibit specific gene expression profiles that distinguish them from those in superficial layers ${ }^{18}$. Alternatively, Shh activity in mature astrocytes may occur

47 independently of their lineage, suggesting that Shh signaling in progenitor cells is uncoupled

48 from its activity in mature cells.

In this study, we performed fate mapping of progenitor cells expressing Gli1 in the

50 postnatal brain to determine whether these cells generate cortical astrocytes and mapped their

51 distribution across cortical layers. We found that a subpopulation of astrocyte progenitor cells

52 in the SVZ express Gli1. Astrocytes within the Gli1 lineage contribute half of the total cortical

53 astrocyte population and are distributed more broadly than those experiencing active Shh

54 signaling in the mature brain. Furthermore, our data show that lineage does not predict Shh

55 activity in mature astrocytes, suggesting that activity of the pathway in progenitor cells is

56 independent from that in mature astrocytes. This may reflect reutilization of the Shh signaling

57 pathway in cells in a manner that is uncoupled from its roles in their development. Taken

58 together, these data demonstrate that Shh signaling identifies a subpopulation of glial

59 progenitor cells that contribute a substantial proportion of protoplasmic astrocytes to the

60 cortex and may have implications for identifying functional heterogeneity of astrocytes.

61 Moreover, these data add to a growing repertoire of cell type-dependent activities orchestrated

62 by Shh signaling. 


\section{Astrocytes in the Gli1 lineage are broadly distributed in the cortex}

65 We first examined the expression of Gli1 in the early postnatal mouse cortex in Gli $1^{\text {nlacz/+ }}$ mice carrying a nuclear lacZ in the Gli1 locus ${ }^{19}$. At postnatal day 0 (P0), there was a pronounced population of Gli1-expressing cells in the ventral subventricular zone (SVZ), corresponding to the population of SVZ precursors that generate deep granule neurons and periglomerular cells in the olfactory bulb ${ }^{15,20,21}$; (Figure 1). Gli1-expressing cells were also observed in the dorsolateral corner of the SVZ (dISVZ), within the postnatal germinal zone of cortical

71 astrocytes ${ }^{8}$, and in a region inferior to the white matter overlying the ventricles (Figure 1). Few

72 cells were observed in the cortex at this age (Figure 1). We quantified and mapped the

73 distribution of $\beta$ Gal-labeled cells within the dISVZ and cortex and found that the cortex

74 harbored relatively few Gli1-expressing cells at PO compared to the dISVZ (Figure 1).

To investigate whether Gli1-expressing precursors observed at P0 generate astrocytes and/or oligodendrocytes, we performed fate mapping in Gli ${ }^{\mathrm{CreER} /+}$ mice $^{14}$ carrying the Ai14

77 tdTomato (tdTom) reporter ${ }^{22}\left(\right.$ Gli $^{\text {CreER/+} ; A i 14) . ~ C r e-m e d i a t e d ~ r e c o m b i n a t i o n ~ p r o m o t e s ~}$ expression of tdTom that is both permanent and heritable. We marked Gli1-expressing precursors by administering tamoxifen to Gli ${ }^{\mathrm{CreER/+}} ; \mathrm{Ai} 14$ mice at $\mathrm{PO}$ and analyzed the distribution of tdTom at various postnatal ages. One day after tamoxifen (P1), there was a

81 substantial population of marked cells in the cortex (Figure 1). At P3, there was a dramatic

82 expansion of marked cells (Figure 1), suggesting extensive proliferation between P1 and P3.

83 We also observed many residual radial glial fibers and cells with transitional morphologies,

84 consistent with radial glia undergoing transformation into multipolar astrocytes (Supplemental

85 Fig. 1). These cells co-express vimentin, a marker of radial glia (Supplemental Fig. 1). There 
87 marked at P0 correspond to actively dividing glial progenitor cells. There was no further

88 expansion in the number of marked cells between P7 and P14, suggesting the cessation of

89 proliferation of cells marked at birth. To confirm the observed expansion was due to

90 proliferation, we administered BrdU to mice at P0 approximately 12 hours after tamoxifen, to

91 ensure sufficient Cre-mediated recombination prior to incorporation of BrdU. We analyzed

92 tissues at P14 and found that $49 \%$ of marked cells in the cortex are co-labeled with BrdU (-

93 Figure 1), indicating that Gli1 cells marked at P0 are dividing. Conversely, $54 \%$ of BrdU

94 labeled cells co-expressed tdTom, suggesting that only a fraction of the proliferating glial

95 precursor cell population residing in the cortex at P0 express Gli1 (Figure 1). Notably, the

distribution of marked cells at these postnatal ages was remarkably distinct from that observed

97 in the adult. Whereas tamoxifen administered to adult mice (>P90) produces a distinctive pattern of marked cells found predominantly in layers IV and $\mathrm{V}^{16}$, marking Gli1-expressing cells at PO produces a broad distribution of marked cells in the cortex with no laminar specificity

(Figure 1). These data show that cells within the Gli1 lineage divide rapidly during the first week after birth, and occupy a much broader laminar distribution than that observed in mature astrocytes.

The postnatal ventricular-subventricular zone (V-SVZ) harbors a dorsal domain of Gli1

104 expressing radial glia that generates oligodendrocytes which populate the white matter ${ }^{13}$. Our

105 results indicate that cells marked at P0 are not restricted to the white matter as we observed a

106 substantial population of marked cells in the cortex as early as 1 day after tamoxifen. To

107 identify the cell types derived from Gli1-expressing precursors, we performed colocalization

108 analysis of marked cells in the cortex with glial cell-type specific markers at P14. Although a

109 small fraction of marked cells co-expressed the oligodendrocyte-specific marker, CC1 (Figure

110 2), the vast majority of marked cells corresponded to astrocytes (Figure 2). We examined the

111 morphologies of marked cells and found that at early time points, marked cells showed simple 
112 morphologies, with one or two processes, consistent with an immature phenotype (Figure 2).

113 Over time, marked cells developed an increasingly complex morphology, extending multiple

114 processes. By P14, cells exhibited a bushy appearance, with many fine branchlets in addition

115 to several major primary branches, consistent with an astrocyte morphology (Figure 2). Taken

116 together, these data indicate that Shh signaling is not restricted to mature astrocytes, but is

117 active in progenitor cells in the neonatal dISVZ that gives rise to a substantial proportion of

118 cortical astrocytes. Our data further show that astrocytes within the Gli1 lineage occupy all

119 layers of the cortex.

120 Whether a Gli1-expressing bi-potent glial progenitor cell generates both cortical

121 astrocytes and white matter oligodendrocytes, or whether these reflect two distinct

122 populations that share Shh activity is not known. Notably, we observed substantial labeling of

123 tdTom in the white matter overlying the ventricles in tissues marked as late as P7 (Figures 1

124 and 4). Further experiments are required to determine whether these cells share a common

125 progenitor or represent two separate populations.

\section{Gli1 progenitors generate a subpopulation of cortical astrocytes}

We next investigated the extent to which astrocytes in the Gli1 lineage contribute to the

128 total astrocyte population in the mature cortex. We administered tamoxifen at P0 and analyzed

129 the fraction of cells labeled with the pan-astrocytic marker, $\mathrm{S} 100 \beta$, that co-express tdTom at

130 P60. We found that $46 \%$ of astrocytes were co-labeled with tdTom (Figure 3), indicating that

131 astrocytes within the Gli1 lineage make up nearly half of the total cortical astrocyte population.

132 This suggests that the cortex harbors a mixed population of astrocytes from different lineages.

133 These data further suggest that Gli1-expressing astrocyte progenitor cells comprise a

134 subpopulation of the total pool of astrocyte progenitors. Alternatively, these data could reflect

135 limitations of tamoxifen-dependent Cre-mediated recombination. To rule out the possibility 
that recombination was inefficient within the Gli1-expressing progenitor population, we

137 examined the dISVZ of Gli $1^{\text {nlacz/+ }}$ mice, enabling us to identify cells actively expressing Gli1

138 independent of the requirement for recombination. We identified the pool of astrocyte

139 progenitors at P0 by expression Sox9 and found that 31\% co-expressed Gli1 (Figure 3),

140 consistent with the idea that progenitor cells expressing Gli1 comprise a subpopulation of

141 astrocyte progenitors in the P0 dISVZ. This was confirmed with a second astrocyte progenitor

142 marker, BLBP, in which we found that many BLBP labeled cells were Gli1 negative (Figure 3).

143 Taken together, these data suggest that the astrocyte progenitor pool in the dISVZ at P0 is

144 comprised of a molecularly distinct subpopulation that can be defined by Shh signaling.

\section{Shh signaling in the cortex is upregulated during postnatal development}

146 Our fate mapping studies showed a differential distribution of cells marked in the

147 neonatal compared to the adult brain. Most notably, genetic marking at P0 produces many

148 labeled cells localized to superficial layers of the cortex, in contrast to adulthood in which

149 marked cells are found predominantly in deep layers (Figure 4). We next investigated the age

150 at which the distinctive laminar pattern of Shh signaling in the cortex emerges. We

151 administered tamoxifen over three consecutive days at various ages during postnatal

152 development. Tissues were analyzed two weeks after the initial tamoxifen dose, with the

153 exception of P7 animals which were analyzed at one and three weeks after tamoxifen. There

154 was no difference between these chase periods and the data were pooled for P7. As with P0,

155 the vast majority of marked cells labeled at these postnatal ages were subsequently identified

156 as astrocytes (Table 1). Analysis of the fraction of marked cells in each layer shows that cells

157 marked at P0 are distributed throughout all layers, whereas the vast majority of cells marked in

158 the adult brain are found in deep layers (Figure 4). At P3, the distribution of marked cells

159 remains broad throughout all cortical layers. By P7, fewer marked cells are found in the upper 
cortical layers than in tissues marked at younger ages (Figure 4). However, by P14 marked cells are found predominantly in deep layers and are largely absent from superficial layers, a pattern that was consistent with that observed at P28 and beyond (Figure 4). This was associated with a concomitant reduction in the fraction of astrocytes, identified by $S 100 \beta$, that are marked at these ages (Figure 4). At P0, 49\% of astrocytes across all layers are marked,

165 but by P3, that proportion declined to 32\%, though this was not significant (Figure 4). At P7, the fraction of marked astrocytes declined significantly from P0 to $23 \%$ This fraction remained

167 steady at P14 and P28 (21\% and 24\%, respectively; 15\%, Figure 4). There was a further

168 modest reduction to $15 \%$ in adults, though this was not significant (Figure 4). Double labeling with BrdU administered 12 hours after tamoxifen shows that the fraction of marked cells that are dividing declines dramatically over the first postnatal week. At P0, the fraction of marked

171 cells double labeled with BrdU was 49\%, whereas at P3, that fraction was significantly reduced 172 to $33 \%$. By P7, nearly all marked cells were postmitotic as only $3 \%$ of tdTom cells co-labeled 173 with BrdU (Figure 4). This suggests that astrocytes within the Gli1 lineage are generated 174 predominantly during the first few days after birth.

176 first postnatal week, analysis of active Shh signaling the cortex in Gli1 ${ }^{\text {lacz/+ }}$ mice shows a

177 progressive increase over the first two postnatal weeks (Figure 4). Stereological quantification 178 of the number of $\beta$ Gal labeled cells in the cortex showed a significant increase in the number of 179 cells at P14 compared to P3 (Figure 4). There was no difference between P14 and the adult 180 (>P90) cortex (Figure 4), suggesting that Shh signaling in the cortex stabilizes by the second 181 postnatal week. Despite the increase in the number of cells exhibiting Shh activity between P7 182 and adulthood, few cells marked at P7 are proliferating (Figure 4), arguing against the 183 possibility that the increase in Gli1-expressing cells between P7 and adulthood is due to 
184 proliferation of immature progenitor cells. These data suggest that Shh signaling in astrocyte

185 progenitor cells residing in the SVZ is transient and is lost as cells migrate into the cortex and

186 undergo maturation. In parallel, Shh activity in the cortex is low at birth, but increases during

187 postnatal development in a subpopulation of mature, postmitotic astrocytes found mostly in

188 deep layers, coincident with the localization of Shh-expressing neurons in layer $\mathrm{V}^{17,23}$. Taken

189 together, these data suggest that Shh signaling in mature cortical astrocytes operates

190 independently of that in SVZ progenitor cells.

\section{Lineage does not predict Shh activity in mature astrocytes}

192 We next examined whether Shh signaling in mature astrocytes is restricted to those within the

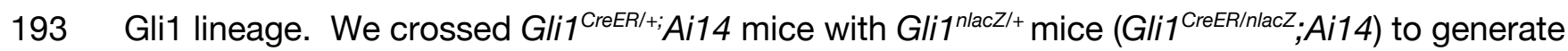

194 mice in which we could distinguish between temporally distinct populations of cells expressing

195 Gli1. We reasoned that because tamoxifen administered at P0 will indelibly mark progenitor

196 cells expressing Gli1 and their progeny, whereas $\beta$ Gal-expressing cells would reflect Gli1

197 activity at the conclusion of the experiment, this approach would enable us to identify

198 individual cells showing differential Gli1 activity at two different time points within a single

199 mouse. While this effectively produces a Gli1 null mouse, Gli1 is not required for Shh signaling

200 during development and Gli1 null mice show no developmental or behavioral deficits ${ }^{19}$. We

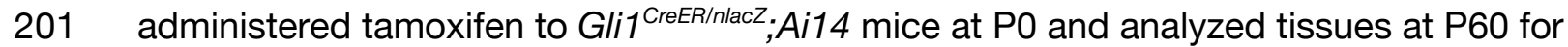

202 colocalization of the tdTom and $\beta$ Gal reporter proteins. Although cells marked at P0 are found

203 throughout all cortical layers, this analysis was restricted to deeper layers where $\beta$ Gal-

204 expressing cells are mostly found. At P60, a large proportion (67\%) of tdTom-labeled cells did

205 not co-express $\beta$ Gal (Figure 5), suggesting that in a substantial fraction of astrocytes in the

206 Gli1 lineage, Shh signaling is downregulated sometime after P0. The remaining fraction of

207 tdTom-labeled cells were double labeled with $\beta$ Gal (Figure 5), suggesting either persistent Shh 
activity since birth or re-induction of Gli1 in the same cell. Interestingly, $40 \%$ of $\beta$ Gal-labeled

209 cells did not co-express tdTom (Figure 5), suggesting active Shh signaling in mature

210 astrocytes that are not within the Gli1 lineage. Taken together, these data suggest that Shh

211 signaling in differentiated astrocytes reflects recurrence of Shh activity in mature cells that is

212 independent of pathway activity in postnatal progenitor cells residing in the SVZ.

213 DISCUSSION

$214 \quad$ Using genetic fate mapping together with a direct reporter of Shh activity, we identified

215 Shh signaling in progenitor cells that generate a substantial proportion of cortical astrocytes.

216 Shh activity is transient and is lost as postnatal development proceeds. Astrocytes within the

217 Gli1 lineage contribute half of all cortical astrocytes, consistent with the observation that Shh is

218 active in a subpopulation of astrocyte progenitors in the neonatal brain. Astrocytes within the

219 Gli1 lineage are distributed broadly across superficial and deep cortical layers, in contrast to

220 the laminar pattern of Shh signaling in the mature cortex. Finally, our data show that lineage

221 does not predict Shh activity in mature astrocytes, suggesting that the role of Shh signaling in

222 mature astrocytes is independent of its role in progenitor cells.

The diversity of mature astrocyte populations has gained increasing recognition, but

224 how such diversity is achieved is not well understood. One mechanism by which the CNS can

225 achieve such diversity is through the production of cells from distinct progenitor cell lineages.

226 In the embryonic spinal cord and forebrain, astrocyte progenitor cells residing in molecularly

227 and anatomically distinct domains produce regionally specified astrocytes that occupy the

228 overlying territory defined by radial glial trajectories ${ }^{24,25}$. Here, we show that Shh signaling

229 operates in a subpopulation of astrocyte progenitor cells residing within the postnatal SVZ. 
232 single domain. The extent to which lineage confers distinct molecular or functional

233 characteristics in astrocytes is not known. The postnatal V-SVZ harbors neural stem cell

234 populations with distinct positional identities that generate specific subpopulations of olfactory

235 bulb interneurons ${ }^{26-28}$. Whether astrocytes within the Gli1 lineage represent a functionally

236 distinct class of cells is unknown and should be the basis of further studies.

One limitation of our study is the requirement for tamoxifen to trigger Cre-mediated

238 recombination. It is possible that the dose and timing of tamoxifen may be insufficient to label

239 all progenitor cells expressing Gli1. However in Gli1 ${ }^{\text {nlacz/+ }}$ mice in which expression of the

240 reporter protein is directly regulated by Gli1 activity, we found that $30 \%$ of progenitor cells

241 express Gli1 in these mice. Given this proportion, our observation that half of the astrocyte

242 population in adult is marked is somewhat lower than might be expected. One possibility is

243 that astrocytes undergo developmental elimination. Indeed, astrocytes in the retina are

244 engulfed by microglia during early postnatal development ${ }^{29}$, and the authors further report a

245 developmental decline in cortical astrocytes in the adult. Such elimination of cells during

246 postnatal maturation could underlie our observations and suggests that Gli1-expressing

247 progenitors generate an even larger fraction of cortical astrocytes than we observed in this

248 study. Nevertheless, our observation that only a fraction of astrocyte progenitor cells express

249 Gli1 at P0 is consistent with the idea that Shh signaling identifies a defined subpopulation of

250 astrocyte progenitor cells. It is possible that the astrocyte progenitor pool at this age may

251 reflect cells in different stages of development. It will be interesting to explore whether timing

252 cooperates with molecular signaling to confer specific functional properties upon astrocytes

253 derived from molecularly and temporally distinct pools of progenitor cells.

254 Astrocytes are derived from three sources, radial glia, progenitors in the SVZ, and local

255 proliferation of astrocytes in the $\operatorname{cortex}^{7,8,12,30}$. Our data show that cells expressing Gli1 at P0

256 are found in substantially greater numbers in the dISVZ, with relatively fewer cells in the cortex. 
257 In addition, we observed many radial glial processes as well as cells with transitional unipolar

258 or bipolar morphologies, consistent with the transformation of radial glia into multipolar

259 astrocytes. These data suggest that Shh is operating in radial glial and SVZ progenitors,

260 although we cannot rule out the possibility that Shh signaling is also acting on proliferating

261 cells in the cortex that are expanding the population of Gli1 lineage astrocytes. However if this

262 is the case, then their proliferation capacity appears to be exhausted before P7, as only $3 \%$ of

263 marked cells in the cortex are proliferating in animals receiving both tamoxifen and BrdU at this

264 age. This argues against the possibility of local proliferation, and instead suggests that as

265 progenitor cells in the Gli1 lineage migrate away from the SVZ and into the cortex, they lose

266 sensitivity to Shh signaling. This may reflect the availability of SHH to astrocyte progenitors

267 and mature astrocytes and suggests that there may be differential sources of ligand to these

268 cell populations. There are several potential sources of $\mathrm{SHH}$ in the neonatal and postnatal

269 brain which include ventral forebrain neurons ${ }^{15}$, cortical neurons ${ }^{17,31}$, epithelial cells ${ }^{32}$, and the

$270 \mathrm{CSF}^{33,34}$. Because high levels of $\mathrm{SHH}$ are required to stimulate Gli1 expression ${ }^{19}$, our data

271 suggest that Gli1-expressing progenitors are migrating away from their neonatal source of

$272 \mathrm{SHH}$. Furthermore, our data suggest that astrocytes within the Gli1 lineage are generated

273 predominantly during the first few days after birth and that Shh activity in astrocyte progenitor

274 cells is transient and declines as astrocytes undergo maturation.

275 Intriguingly, our fate mapping studies show that while the fraction of astrocytes in the

276 cortex that are marked declines progressively between P0 and P14, the number of cells

277 showing active Shh signaling increases progressively during this time. Shh levels in the

278 postnatal cortex are developmentally regulated and are low at birth but increases progressively

279 until its peak at $\mathrm{P} 14^{23}$. This suggests that as early postnatal development proceeds, Shh

280 activity in the cortex reflects a recurrence of the pathway in differentiated cells, independently

281 of activity in SVZ progenitor cells. Moreover, lineage does not predict Shh activity in mature 
astrocytes, as cells that were not marked at PO nevertheless exhibit Shh activity in adulthood.

283 Consistent with this, nearly all the components of the Shh signaling pathway, including the 284 receptor Patched (Ptc), and the transcriptional effectors Gli2 and Gli3 are found in a greater number of astrocytes than are actively expressing Gli $1^{3}$, suggesting that many cells possess the machinery to transduce $\mathrm{SHH}$. This may reflect dynamic activity of the pathway and may be one mechanism by which neurons can recruit neighboring astrocytes depending on local

288 needs.

Shh signaling regulates a diverse repertoire of cellular activities in multiple cell types,

290 including neural stem and progenitor cells, neurons and astrocytes ${ }^{35}$. It is likely that the

291 molecular programs initiated by Shh activity in astrocyte progenitors and mature astrocytes

292 confer distinct functional characteristics that are cell-type dependent. This application of a

293 single molecular pathway in cell type dependent ways reflects the pleiotropic nature of Shh

294 signaling and demonstrates the remarkable capacity of the pathway to be deployed in a broad 295 array of cellular activities.

298 Animals. The following transgenic mouse lines were used: Gli $1^{\text {nLacZ/+,19 }}$, Gli $1^{\text {CreER/+,14}}$, and $299 R 26^{t d T o m / t d T o m}(A i 14)^{36}$. Animals were maintained on a 12h light/dark cycle and given access to 300 food and water ad libitum. All experiments were conducted in accordance with the Drexel 301 University Institute for Animal Care and Use Committee. Male and female animals between 302 postnatal day (P) 0 and P60 were used.

303 Tamoxifen. Tamoxifen (Sigma, T5648-1G) was diluted to a final concentration of $5 \mathrm{mg} / \mathrm{ml}$ or $30410 \mathrm{mg} / \mathrm{mL}$ in corn oil. P0 Gli ${ }^{\mathrm{CreER} /+} ;$ Ai14 mice received 50mg/kg tamoxifen by intragastric 305 injection for 1 or 3 days consecutively and tissue was harvested at various indicated time 
points. All other Gli1 ${ }^{\mathrm{CreER} /+} ;$ Ai14 mice received $100 \mathrm{mg} / \mathrm{kg}$ of tamoxifen by intragastric injection

307 (P3), subcutaneous injection (P7), or oral gavage (P14 and above) for 1 or 3 days consecutively, and tissue was harvested at indicated time points. For adult comparisons, Gli1 ${ }^{\mathrm{CreER} /+} ;$ Ai14 mice greater than 2 months old received $250 \mathrm{mg} / \mathrm{kg}$ of tamoxifen by oral gavage for 1 or 3 days consecutively, and tissue was harvested 2 weeks later, unless otherwise noted.

311 BrdU. BrdU (Sigma, B9285-1G) was dissolved in $0.007 \mathrm{~N} \mathrm{NaOH}$ in sterile saline and 312 administered via intraperitoneal (i.p.) injection. For long term experiments, mice received $31350 \mathrm{mg} / \mathrm{kg}$ in mice ages P0 to P14 or $200 \mathrm{mg} / \mathrm{kg}$ at P28 and older, at $6-24$ hours after 314 tamoxifen.

315 Perfusion and histology. Animals were given an i.p. injection of a

316 Ketamine/Xylazene/Acepromazine cocktail and transcardially perfused with 10mM PBS

317 followed by $4 \%$ paraformaldehyde solution. Brains were dissected and post-fixed in $4 \%$

318 paraformaldehyde for 4-6 hours followed by cryoprotection in $30 \%$ sucrose and stored at $4^{\circ} \mathrm{C}$

319 for at least 48 hours or until ready for sectioning. For P0 and P3, tissue was embedded in OCT

320 medium after cryoprotection and stored at $-20^{\circ} \mathrm{C}$ until sectioning. This tissue was sectioned by

321 cryostat at $16-20 \mu \mathrm{m}$ onto coated slides and stored at $-80^{\circ} \mathrm{C}$, protected from air and light. All

322 other tissue was sectioned by cryostat at $40 \mu \mathrm{m}$ and stored at $4^{\circ} \mathrm{C}$ in TBS with $0.05 \%$ sodium

323 azide. Immunohistochemistry was performed on every $12^{\text {th }}$ section both for free floating and

324 slide-mounted tissues using the following primary antibodies for fluorescence: rabbit anti- $\beta$ gal

325 (1:1k/1:10kMP Biomedicals), chicken anti-ßgal (1:1k, Abcam), mouse anti-BLBP (1:1k, Abcam), 326 sheep anti-BrdU (1:500, Maine Biotechnology Services), mouse anti-CC1 (1:1k, Calbiochem), 327 rabbit anti-RFP (1:500, MBLI), rabbit anti-S100ß (1:1k, DAKO), goat anti-Sox9 (1:1000 R\&D), 328 and chicken anti-Vimentin (1:1k, Invitrogen). For BrdU staining, tissue was pre-incubated in $2 \mathrm{~N}$ $329 \mathrm{HCl}$ for 30 minutes and neutralized with $0.1 \mathrm{M}$ TBS before incubation in block and primary 
antibody. Fluorescent labeling was achieved using species-specific AlexaFluor-tagged secondary antibodies, Alexa Fluor 488, Alexa Fluor 568, or Alexa Fluor 647 (1:1k, Life

332 Technologies), followed by counterstaining with DAPI (1:36k, Life Technologies). For brightfield

333 immunostaining, tissues were quenched in TBS with $0.3 \% \mathrm{H}_{2} \mathrm{O}_{2}$ and $30 \%$ methanol for 30

334 minutes prior to incubation in block and primary antibody. The following antibodies were used:

335 rabbit anti-ßgal (1:40K, MP Biomedicals) and rabbit anti-RFP (1:500, MBLI). For brightfield

336 staining, species-specific biotinylated secondary antibodies (Vector) were used at 1:400

337 followed by incubation in avidin-biotin complex ( $\mathrm{BBC}$, Vector). Visualization was achieved using

338 3'-3 diaminobezedine (DAB, Vector) as the developing agent. 4\% paraformaldehyde post-fix

339 was applied to all slide-mounted tissue for 30 minutes prior to staining.

340 Microscopy. Stained sections were examined and imaged in brightfield and fluorescence using

341 an upright microscope (Zeiss) and Stereolnvestigator software (MBF Biosciences). Confocal

342 images were obtained on an inverted microscope (Leica) using LAS X software. Single-cell

343 analysis of co-labeling was evaluated on double-stained immunofluorescent tissues by taking

344 confocal sections with a 1um slice distance with $20 x, 40 x$ oil or $60 x$ oil objective. For each

345 cortex sampled, multiple $z$ stacks were collected from 3-5 sections within the anterior and

346 posterior boundaries defined by figure 22 and figure 44, respectively from Paxinos and Franklin

347 (2013).

348 Cell quantification. Stereological estimates of the total number of cells was performed in 349 tissues stained for brightfield microscopy. The cortex was analyzed in a series of 6-8 sections,

350 sampled every $480 \mu \mathrm{m}$, and bounded by the midline dorsally and the end of the external

351 capsule ventrally. Anterior and posterior boundaries were defined figure 22 and figure 44,

352 respectively from Paxinos and Franklin ${ }^{37}$. We used a modified optical fractionator and

353 stereological image analysis software (Stereolnvestigator, MBF Bioscience) operating a 
354 computer-driven stage attached to an upright microscope (Zeiss). The cortical area to be

355 analyzed was outlined at low magnification, and counting frames were selected at random by

356 the image analysis software. Cells were counted using a 40x objective and DIC optics. A target

357 cell count of 300 cells was used to define scan grid and counting frame sizes, with a $2 \mu \mathrm{m}$

358 guard zone. For all analyses, only cells with a clear and distinct labeled cell body were

359 analyzed. Analysis of cells in the cortex and dISVZ was performed on tissues stained for

360 brightfield microscopy. Each region was traced in Neurolucida (MBF Bioscience) at 5x and

361 individual $\beta$ gal-labeled cells were mapped with separate markers in each region at 40x using

362 DIC optics.

363 Statistics. All statistical analyses were performed using Prism 8 (GraphPad).

364 Acknowledgements

365 The authors are grateful to Dr. Corey Harwell (Harvard Medical School) for helpful discussions 366 and critical feedback. 


\section{REFERENCES}

$1 \quad$ Fuccillo, M., Joyner, A. L. \& Fishell, G. Morphogen to mitogen: the multiple roles of hedgehog signalling in vertebrate neural development. Nature Reviews Neuroscience 7, 772-783, doi:10.1038/nrn1990 (2006).

2 Farmer, W. T. \& Murai, K. Resolving Astrocyte Heterogeneity in the CNS. Front Cell Neurosci 11, 300, doi:10.3389/fncel.2017.00300 (2017).

3 Garcia, A. D. R., Petrova, R., Eng, L. \& Joyner, A. L. Sonic Hedgehog Regulates Discrete Populations of Astrocytes in the Adult Mouse Forebrain. The Journal of neuroscience : the official journal of the Society for Neuroscience 30, 13597-13608, doi:10.1523/JNEUROSCI.0830-10.2010 (2010).

4 Wallace, V. A. \& Raff, M. C. A role for Sonic hedgehog in axon-to-astrocyte signalling in the rodent optic nerve. Development 126, 2901-2909 (1999).

5 Agius, E. et al. Converse control of oligodendrocyte and astrocyte lineage development by Sonic hedgehog in the chick spinal cord. Developmental Biology 270, 308-321, doi:10.1016/j.ydbio.2004.02.015 (2004).

6 Sauvageot, C. M. \& Stiles, C. D. Molecular mechanisms controlling cortical gliogenesis. Curr Opin Neurobiol 12, 244-249, doi:10.1016/s0959-4388(02)00322-7 (2002).

7 Levison, S. W., Chuang, C., Abramson, B. J. \& Goldman, J. E. The migrational patterns and developmental fates of glial precursors in the rat subventricular zone are temporally regulated. Development 119, 611-622 (1993).

8 Levison, S. W. \& Goldman, J. E. Both oligodendrocytes and astrocytes develop from progenitors in the subventricular zone of postnatal rat forebrain. Neuron 10, 201-212, doi:10.1016/0896-6273(93)90311-e (1993).

9 Misson, J. P., Austin, C. P., Takahashi, T., Cepko, C. L. \& Caviness, V. S., Jr. The alignment of migrating neural cells in relation to the murine neopallial radial glial fiber system. Cereb Cortex 1, 221-229, doi:10.1093/cercor/1.3.221 (1991).

10 Noctor, S. C., Flint, A. C., Weissman, T. A., Dammerman, R. S. \& Kriegstein, A. R. Neurons derived from radial glial cells establish radial units in neocortex. Nature 409, 714-720, doi:10.1038/35055553 (2001).

11 Schmechel, D. E. \& Rakic, P. A Golgi study of radial glial cells in developing monkey telencephalon: morphogenesis and transformation into astrocytes. Anat Embryol (Berl) 156, 115-152, doi:10.1007/BF00300010 (1979).

12 Ge, W. P., Miyawaki, A., Gage, F. H., Jan, Y. N. \& Jan, L. Y. Local generation of glia is a major astrocyte source in postnatal cortex. Nature 484, 376-380, doi:10.1038/nature10959 (2012).

13 Tong, C. K. et al. A Dorsal SHH-Dependent Domain in the V-SVZ Produces Large Numbers of Oligodendroglial Lineage Cells in the Postnatal Brain. Stem Cell Reports 5, 461-470, doi:10.1016/j.stemcr.2015.08.013 (2015).

14 Ahn, S. \& Joyner, A. L. Dynamic changes in the response of cells to positive hedgehog signaling during mouse limb patterning. Cell 118, 505-516, doi:10.1016/j.cell.2004.07.023 (2004). 
15 Ihrie, R. A. et al. Persistent Sonic Hedgehog Signaling in Adult Brain Determines Neural Stem Cell Positional Identity. Neuron 71, 250-262, doi:10.1016/j.neuron.2011.05.018 (2011).

16 Hill, S. A. et al. Sonic hedgehog signaling in astrocytes mediates cell-type-specific synaptic organization. eLife 8, doi:10.7554/eLife.45545 (2019).

17 Harwell, C. C. et al. Sonic hedgehog expression in corticofugal projection neurons directs cortical microcircuit formation. Neuron 73, 1116-1126, doi:10.1016/j.neuron.2012.02.009 (2012).

18 Bayraktar, O. A. et al. Astrocyte layers in the mammalian cerebral cortex revealed by a single-cell in situ transcriptomic map. Nat Neurosci 23, 500-509, doi:10.1038/s41593020-0602-1 (2020).

19 Bai, C. B., Auerbach, W., Lee, J. S., Stephen, D. \& Joyner, A. L. Gli2, but not Gli1, is required for initial Shh signaling and ectopic activation of the Shh pathway.

Development 129, 4753-4761 (2002).

20 Merkle, F. T., Mirzadeh, Z. \& Alvarez-Buylla, A. Mosaic organization of neural stem cells in the adult brain. Science 317, 381-384, doi:10.1126/science.1144914 (2007).

21 Petrova, R., Garcia, A. D. \& Joyner, A. L. Titration of GLI3 repressor activity by sonic hedgehog signaling is critical for maintaining multiple adult neural stem cell and astrocyte functions. J Neurosci 33, 17490-17505, doi:10.1523/JNEUROSCI.204213.2013 (2013).

22 Madisen, L. et al. A toolbox of Cre-dependent optogenetic transgenic mice for lightinduced activation and silencing. Nat Neurosci 15, 793-802, doi:10.1038/nn.3078 (2012).

23 Charytoniuk, D. et al. Sonic Hedgehog signalling in the developing and adult brain. $J$ Physiol Paris 96, 9-16, doi:10.1016/s0928-4257(01)00075-4 (2002).

24 Hochstim, C., Deneen, B., Lukaszewicz, A., Zhou, Q. \& Anderson, D. J. Identification of positionally distinct astrocyte subtypes whose identities are specified by a homeodomain code. Cell 133, 510-522, doi:10.1016/j.cell.2008.02.046 (2008).

25 Tsai, H. H. et al. Regional astrocyte allocation regulates CNS synaptogenesis and repair. Science 337, 358-362, doi:10.1126/science.1222381 (2012).

26 Kohwi, M. et al. A subpopulation of olfactory bulb GABAergic interneurons is derived from Emx1- and Dlx5/6-expressing progenitors. J Neurosci 27, 6878-6891, doi:10.1523/JNEUROSCI.0254-07.2007 (2007).

27 Merkle, F. T. et al. Adult neural stem cells in distinct microdomains generate previously unknown interneuron types. Nat Neurosci 17, 207-214, doi:10.1038/nn.3610 (2014).

28 Young, K. M., Fogarty, M., Kessaris, N. \& Richardson, W. D. Subventricular zone stem cells are heterogeneous with respect to their embryonic origins and neurogenic fates in the adult olfactory bulb. $J$ Neurosci $27,8286-8296$, doi:10.1523/JNEUROSCI.047607.2007 (2007).

29 Puñal, V. M. et al. Large-scale death of retinal astrocytes during normal development is non-apoptotic and implemented by microglia. PLoS Biology 17, e3000492-3000438, doi:10.1371/journal.pbio.3000492 (2019). 
30 Cameron, R. S. \& Rakic, P. Glial cell lineage in the cerebral cortex: a review and synthesis. Glia 4, 124-137, doi:10.1002/glia.440040204 (1991).

31 Komada, M. et al. Hedgehog signaling is involved in development of the neocortex. Development 135, 2717-2727, doi:10.1242/dev.015891 (2008).

32 Choe, Y., Huynh, T. \& Pleasure, S. J. Epithelial cells supply Sonic Hedgehog to the perinatal dentate gyrus via transport by platelets. Elife 4, doi:10.7554/eLife.07834 (2015).

33 Huang, X. et al. Transventricular delivery of Sonic hedgehog is essential to cerebellar ventricular zone development. Proc Natl Acad Sci U S A 107, 8422-8427, doi:10.1073/pnas.0911838107 (2010).

34 Winkler, C. C. et al. The Dorsal Wave of Neocortical Oligodendrogenesis Begins Embryonically and Requires Multiple Sources of Sonic Hedgehog. The Journal of neuroscience : the official journal of the Society for Neuroscience 38, 5237-5250, doi:10.1523/JNEUROSCI.3392-17.2018 (2018).

35 Garcia, A. D. R. et al. The Elegance of Sonic Hedgehog: Emerging Novel Functions for a Classic Morphogen. J Neurosci 38, 9338-9345, doi:10.1523/JNEUROSCI.1662-18.2018 (2018).

36 Madisen, L. et al. A robust and high-throughput Cre reporting and characterization system for the whole mouse brain. Nature Publishing Group 13, 133-140, doi:10.1038/nn.2467 (2009).

37 Paxinos, G. \& Watson, C. Paxino's and Watson's The rat brain in stereotaxic coordinates. Seventh edition. edn, (Elsevier/AP, Academic Press is an imprint of Elsevier, 2014). 
bioRxiv preprint doi: https://doi.org/10.1101/2020.06.17.157065; this version posted June 18, 2020. The copyright holder for this preprint (which was not certified by peer review) is the author/funder, who has granted bioRxiv a license to display the preprint in perpetuity. It is made available under aCC-BY-NC-ND 4.0 International license.
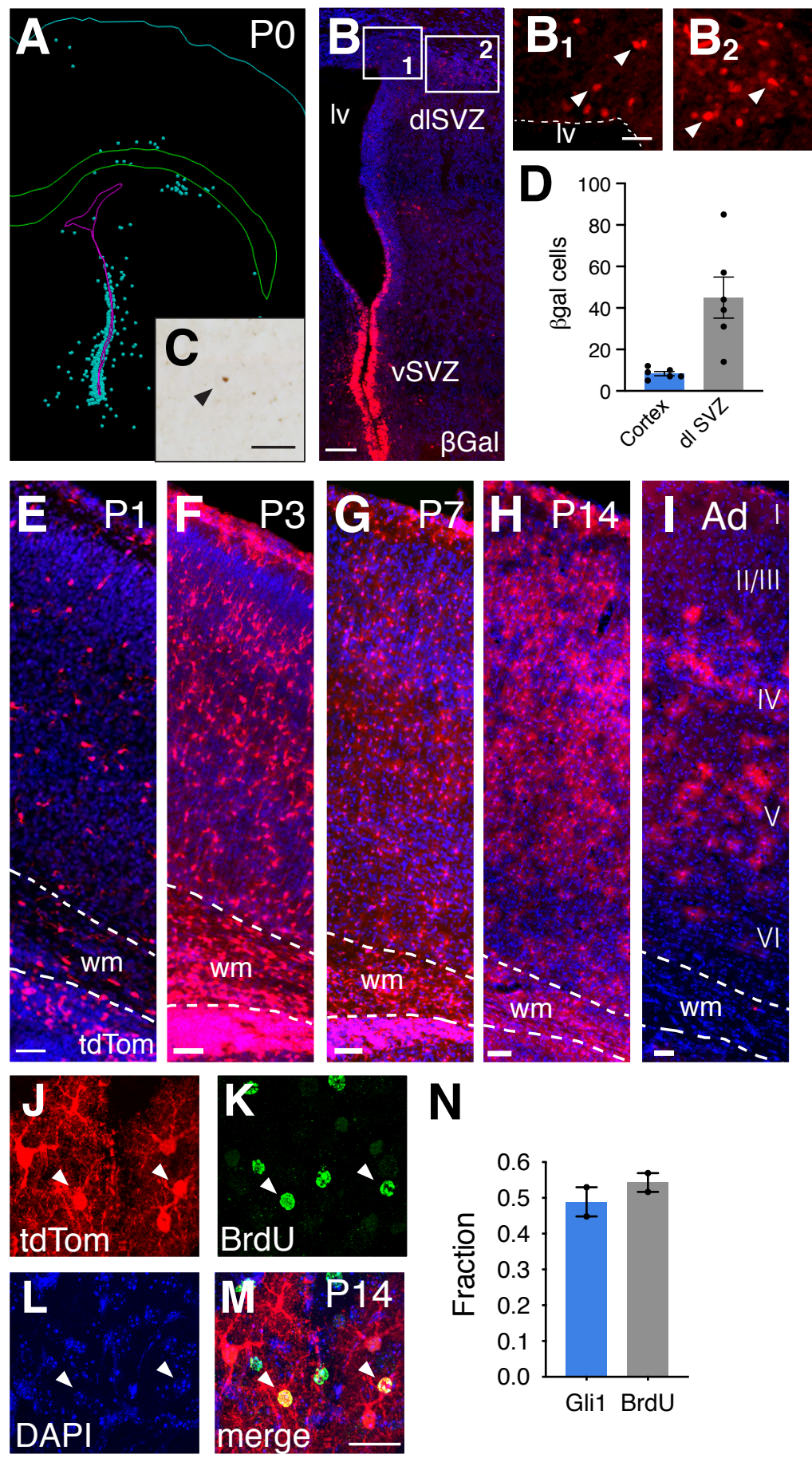

Figure 1. The population of Gli1 cells expands during postnatal development. (A-D) The distribution of $\beta$ Gal-labeled cells in Glit 1/acz/+ tissues at P0. (A) Neurolucida tracing from brightfield immunostained sections. Each dot represents a single cell. (B-C) Immunoflurescent (B) and brightfield (C) staining of $\beta G a l$ at $P 0$ in the SVZ (B) and cortex (C). Insets in $B$ shown in $B_{1}$ and $B_{2}$. Scale bars, $B, 100 \mu m, B_{1}-B_{2}, C, 50 \mu m$. Iv, lateral ventricle, vSVZ, ventral SVZ, dISVZ, dorsolateral SVZ. (D) Quantification of the number of $\beta$ Gal-labeled cells in the cortex and dISVZ at P0. Bars represent mean \pm SEM,

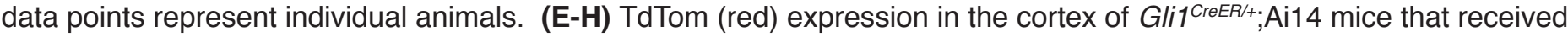
tamoxifen at P0 and analyzed at P1 (E), P3 (F), P7 (G), and P14 (H) shows a much broader distribution of marked cells than that observed in adulthood. (I) Tamoxifen administration in an adult and analyzed 2 weeks later shows marked cells are found predominantly in layers IV and V. Counterstaining with DAPI (blue), wm, white matter. Scale bar, $50 \mu \mathrm{m}$. (J-M) Immunofluorescent staining for BrdU in the cortex of a mouse at P14 that received tamoxifen at P0. Single channel (J-K) and merged (M) max projection images from confocal stacks in the cortex showing colocalization of marked cells (red, J) and BrdU (green, K). Counterstaining with DAPI (blue, L). Arrowheads, double labeled cells. Scale bar, $25 \mu \mathrm{m}$. (N) The fraction of marked cells (Gli1) and dividing cells (BrdU) that are double labeled in mice marked at P0 and analyzd at P14 ( $n=919$ Gli1 and $n=820$ BrdU cells). Bars represent mean \pm SEM, data points represent individual animals. 
bioRxiv preprint doi: https://doi.org/10.1101/2020.06.17.157065; this version posted June 18, 2020. The copyright holder for this preprint (which was not certified by peer review) is the author/funder, who has granted bioRxiv a license to display the preprint in perpetuity. It is made available under aCC-BY-NC-ND 4.0 International license.
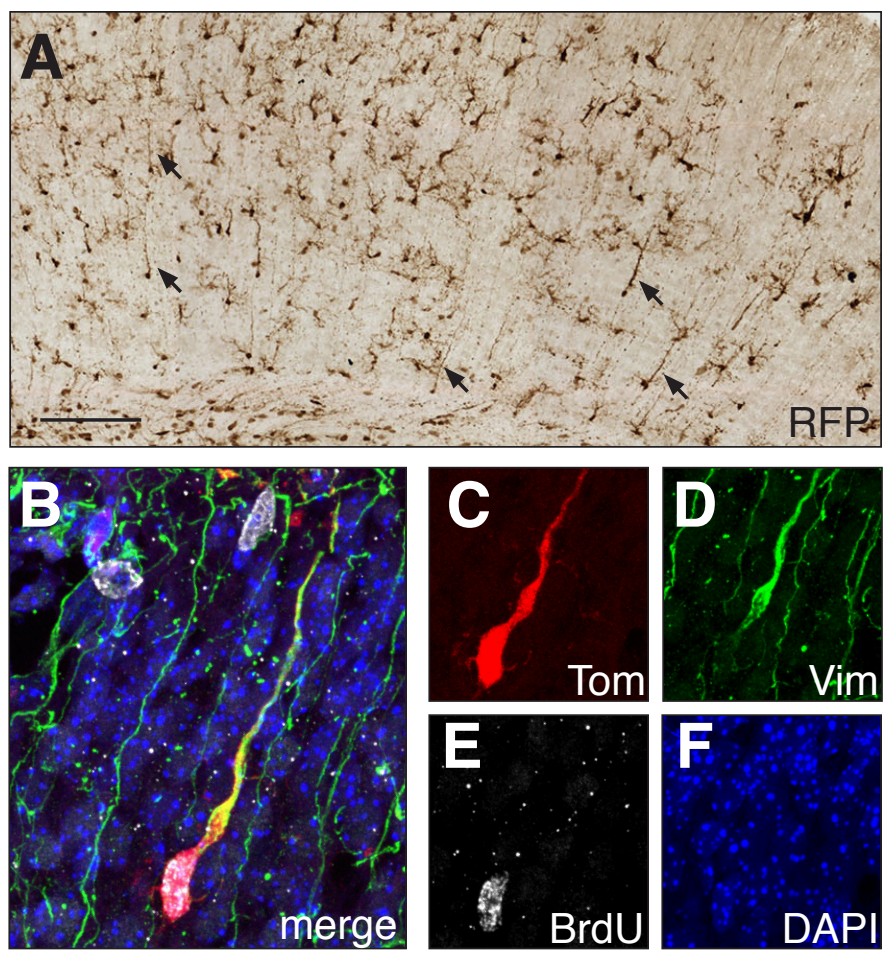

\section{Supplemental Figure 1. Marked cells show} characteristics of transitional radial glia. (A)

Brightfield immunostaining for RFP in the cortex of a mouse at P3 after receiving tamoxifen at P0 showing many cells with transitional morphologies and the appearance of residual radial glial fibers. Scale bar, $25 \mu \mathrm{m}$ (B-F) Colocalization of tdTom (C, red), vimentin ( $D$, green), and BrdU (E, gray) in the cortex of Gli1 ${ }^{\mathrm{CreER} / \text {; }}$ Ai14 mice at P3 after tamoxifen at P0. Merged image in (B), single channel images in C-F. 
bioRxiv preprint doi: https://doi.org/10.1101/2020.06.17.157065; this version posted June 18, 2020. The copyright holder for this preprin (which was not certified by peer review) is the author/funder, who has granted bioRxiv a license to display the preprint in perpetuity. It is made available under aCC-BY-NC-ND 4.0 International license.
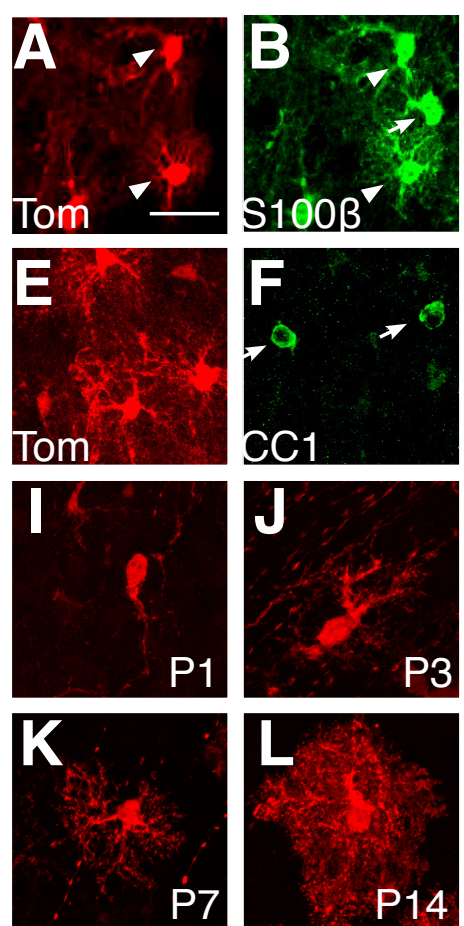

P7
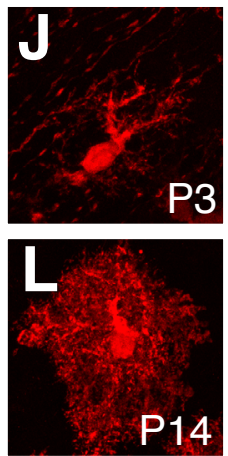

Figure 2. Cells expressing Gli1 at P0 generate cortical astrocytes. (A-H) Single channel (A-C, E-G) and merged images $(D, H)$ from confocal images of tdTom (red) and S100 $(\mathrm{B}$, green) or CC1 ( $\mathrm{F}$, green) in the cortex at P14, following tamoxifen adminstration at PO. Marked cells show colocalization with S100 $\beta$, but not with CC1. Counterstaining with DAPI (C, G, blue). Arrowheads, colocalized cells, arrows, single labeled astrocytes or oligodendrocytes not marked with tom. Scale bar, 25 um. (I-L) Cells marked at P0, and analyzed at P1 (I), P3 (J), P7 $(\mathrm{K})$, and P14 (L) show an increasingly complex morphology consistent with an astrocyte identity as development proceeds. (M) Single cell quantification of the fraction of marked cells at P14 that correspond to astrocytes or oligodendrocytes ( $n=783$ and $n=632$ cells, respectively). Bars represent mean \pm SEM, data points represent individual animals. 
bioRxiv preprint doi: https://doi.org/10.1101/2020.06.17.157065; this version posted June 18, 2020. The copyright holder for this preprint (which was not certified by peer review) is the author/funder, who has granted bioRxiv a license to display the preprint in perpetuity. It is made available under aCC-BY-NC-ND 4.0 International license.
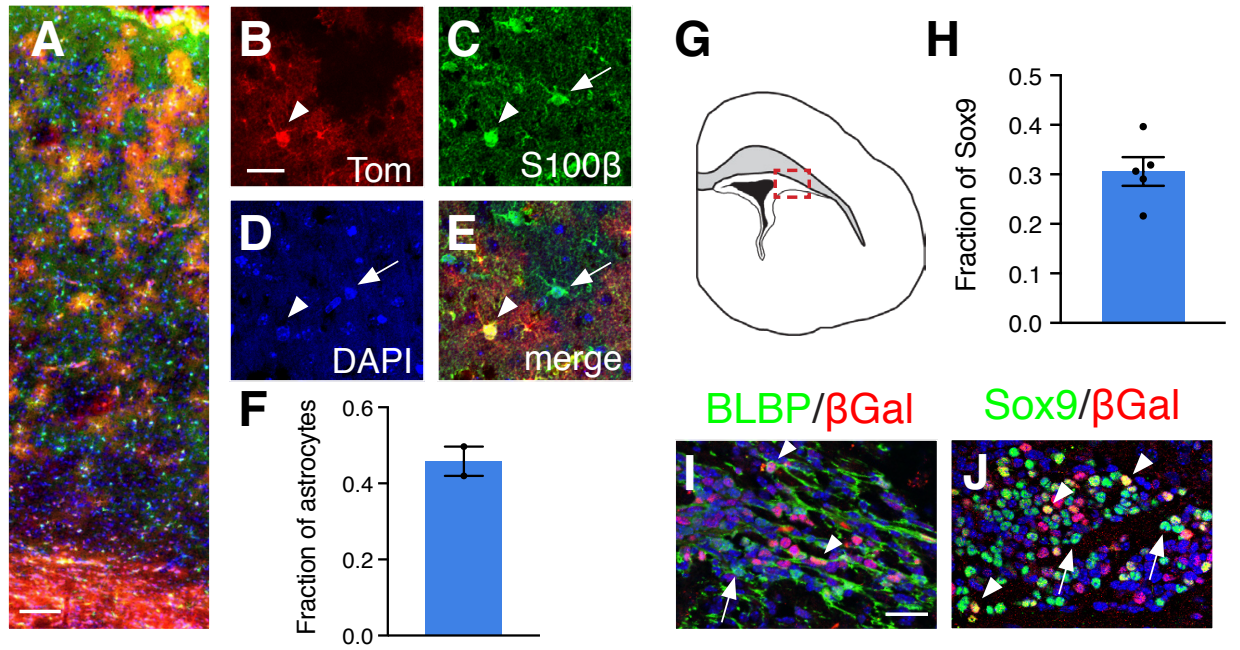

Figure 3. A subpopulation of astrocyte progenitors in the SVZ express

Gli1. (A) Distribution of tdTom (red) in the cortex of adult (P60) Gli1 ${ }^{\text {CreER/+;Ai14 }}$ mouse marked at P0. Scale bar, $100 \mu \mathrm{m}$. (B-E) Single channel, confocal images of tdTom (B, red) and $S 100 \beta$ (C, green) identifying marked cells as astrocytes. Counterstained with DAPI (D, blue). Merged image shown in E. Arrowhead, double labeled cell, arrows, single labeled cells. Scale bar, $25 \mu \mathrm{m}(\mathbf{F})$ The fraction of cortical astrocytes in the Gli1 lineage ( $n=1371$ cells). (G) Tracing of a P0 brain section highlighting the dISVZ (red inset). (H) The fraction of Sox9 labeled cells in the dISVZ co-labeled with $\beta$ Gal at PO ( $n=8358$ cells). (I-J) Double labeling for $\beta$ Gal (red) and the astrocyte progenitor markers BLBP (I, green) or Sox9 (J, green) in the dISVZ of Gli $1^{\text {nlacz/+ }}$ mice at P0. Counterstained with DAPI (blue). Scale bar, $25 \mathrm{um}$. Images taken from red inset shown in G. Bars represent mean \pm SEM, data points represent individual animals. 
bioRxiv preprint doi: https://doi.org/10.1101/2020.06.17.157065; this version posted June 18, 2020. The copyright holder for this preprint (which was not certified by peer review) is the author/funder, who has granted bioRxiv a license to display the preprint in perpetuity. It is made available under aCC-BY-NC-ND 4.0 International license.
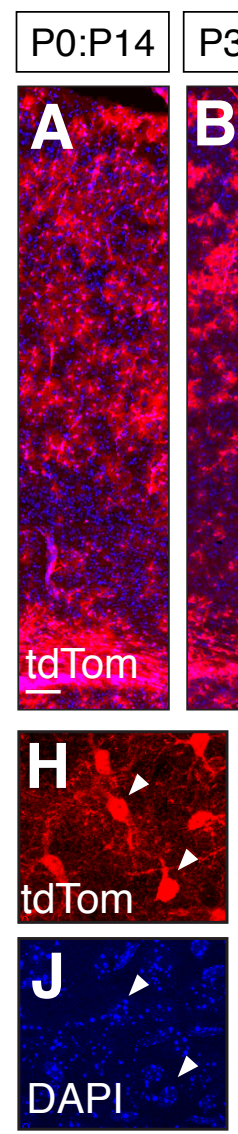
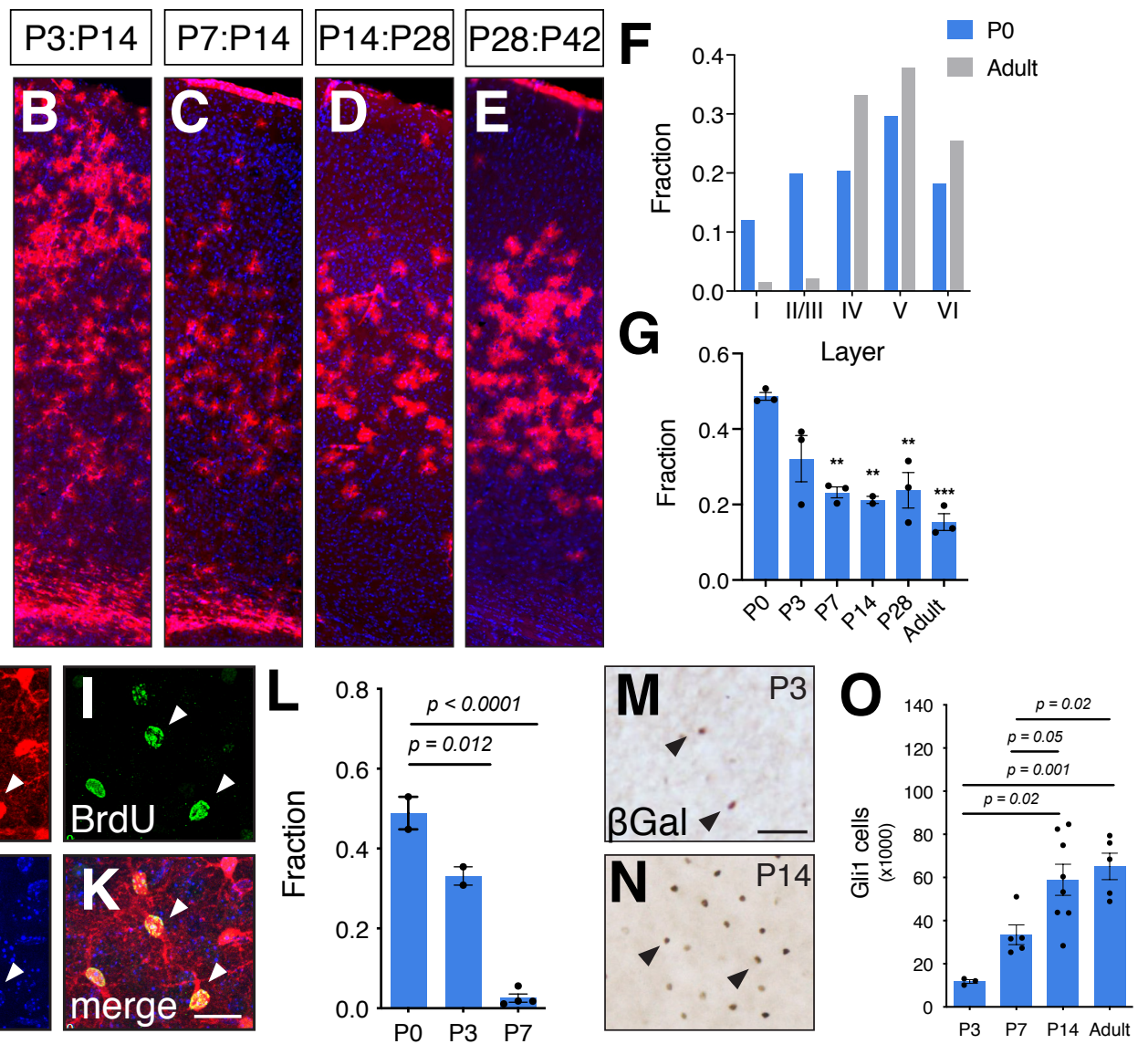

Figure 4. Gli1 cell distribution becomes restricted over postnatal development. (A-E) tdTom (red) expression in Gli1 ${ }^{\mathrm{CreER} /+}$;Ai14 tissues that received tamoxifen at P0 (A), P3 (B), P7 (C), P14 (D), and P28 (E) and analyzed at ages shown. Counterstaining with DAPI (blue). Scale bar, $100 \mu \mathrm{m}$. (F) The fraction of marked cells in each layer in mice that received tamoxifen at PO or in adulthood. (G) The fraction of all astrocytes across all layers that are marked at various ages. Bars represent mean \pm SEM, data points represent individual animals. ${ }^{* *} p<0.005,{ }^{* *} p<0.0005$ compared to P0. (H-K) Single channel, confocal images of tdTom $(\mathrm{H}$, red) and BrdU (I, green) identifying marked cells that are proliferating. Counterstained with DAPI (J, blue). Merged image shown in K. Scale bar, $25 \mu \mathrm{m}$. (L) The fraction of marked cells colabeled with BrdU at P14 in tissues marked at P0, P3 and P7 ( $n=919$ cells, $n=720$ cells, $n=566$ cells, respectively). Bars represent mean $\pm \mathrm{SEM}$, data points represent individual animals. (M-N) Brightfield immunostaining for $\beta$ Gal in Gli1 $1^{\text {lacz/+ }}$ tissues from mice at (M) P3 and (N) P14. Scale bar, $50 \mu \mathrm{m}$. (O) Estimated total number of Gli1-expressing cells derived from stereological quantification of $\beta$ Gal labeled cells in the cortex of Gli1nlacz/+ mice at various ages. Bars represent mean \pm SEM, data points represent individual animals. 
bioRxiv preprint doi: https://doi.org/10.1101/2020.06.17.157065; this version posted June 18, 2020. The copyright holder for this preprint (which was not certified by peer review) is the author/funder, who has granted bioRxiv a license to display the preprint in perpetuity. It is made available under aCC-BY-NC-ND 4.0 International license.

\begin{tabular}{|c|c|}
\hline Age & Astrocytes \\
\hline P0 & $95 \%(783)$ \\
\hline P3 & $99 \%(843)$ \\
\hline P7 & $99 \%(578)$ \\
\hline P14 & $97 \%(348)$ \\
\hline P28 & $98 \%(389)$ \\
\hline Adult & $99 \%(191)$ \\
\hline
\end{tabular}

Table 1. Gli1 cells marked at various ages correspond to astrocyte progenitors or mature astrocytes. Single cell analysis of the identity of marked cells in

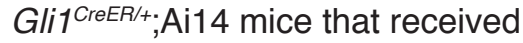
tamoxifen at the ages shown in the left column, and analyzed two weeks later. The fraction of tdTom cells identified as astrocytes by colocalization with $\mathrm{S} 100 \beta$. The number of individual cells analyzed is in parentheses. $n=3-4$ mice for each age. 
bioRxiv preprint doi: https://doi.org/10.1101/2020.06.17.157065; this version posted June 18, 2020. The copyright holder for this preprint (which was not certified by peer review) is the author/funder, who has granted bioRxiv a license to display the preprint in perpetuity. It is made available under aCC-BY-NC-ND 4.0 International license.
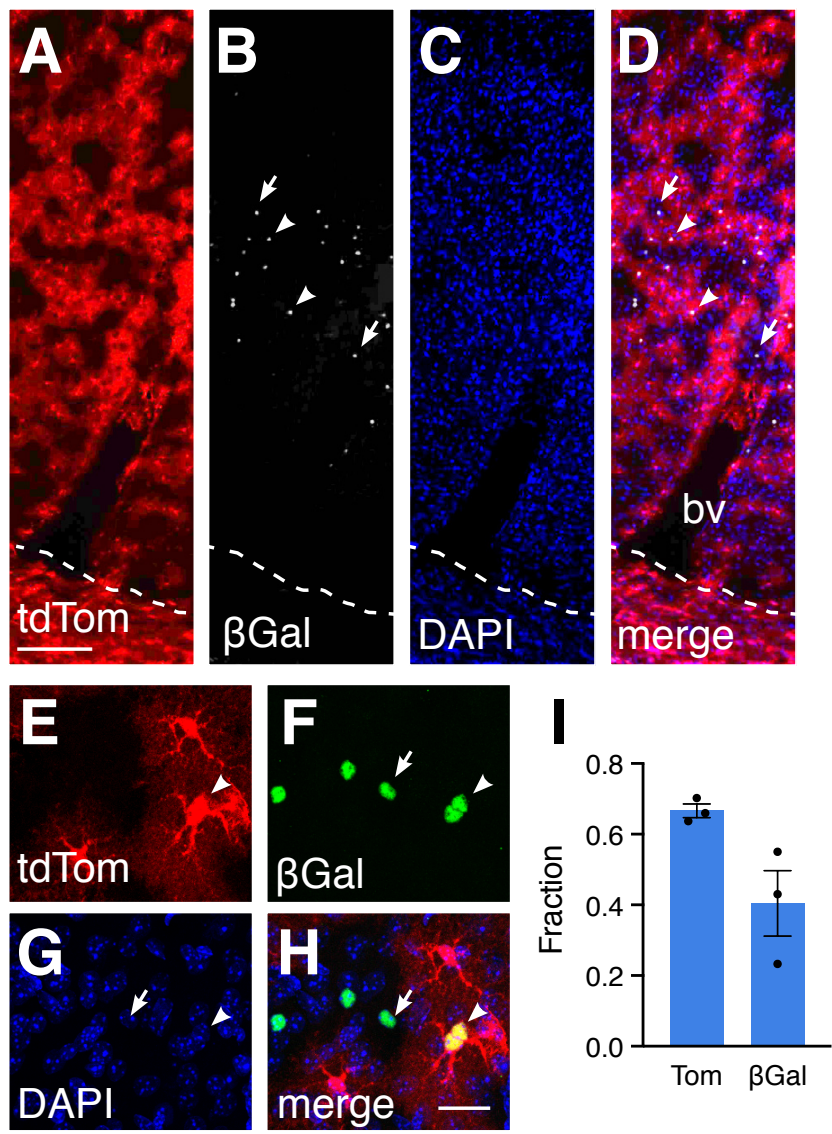

\section{DAPI}

Figure 5. Shh signaling is upregulated in mature astrocytes $(A-H)$ Double labeling for tdTom (A, E, red) and $\beta G a l(B$, grey, $F$, green) in the cortex of a Gli1 CreER/LLacz;Ai14 mouse marked at PO and analyzed at P60. (A-D) Low magnification and (E-H) high magnification confocal images. Counterstained with DAPI (C, G, blue). Dotted line depicts white matter border. Merged images shown in D, H. bv, blood vessel. Arrowheads, colocalized cells, arrows, $\beta$ Gal only cells. (I) The fraction of tdTom or $\beta$ Gal labeled cells that are single labeled ( $n=1454$ and $n=821$ cells, respectively). Bars represent mean \pm SEM, data points represent individual animals. 